\title{
Tendiendo puentes 2.0 entre la historia y el patrimonio marítimo y los usuarios virtuales: el blog y el perfil en Facebook de la cátedra de historia naval
}

\author{
Lorena Martínez Solís, Cátedra de Historia Naval, España \\ Celia Chaín Navarro, Universidad de Murcia, España \\ Juan José Sánchez Baena, Cátedra de Historia Naval ${ }^{1}$, España \\ Fernando Díaz Pérez, Universidad de Murcia, España
}

\begin{abstract}
Resumen: La Cátedra de Historia Naval, impulsada por la Armada Española y la Universidad de Murcia, tiene entre sus objetivos investigar y difundir la Historia y el Patrimonio Naval y Marítimo. Con objeto acercar el conocimiento científico y la información divulgativa versada en esta materia al usuario, nuestra institución no se limita a utilizar los canales clásicos de comunicación como pueden ser los artículos y las monografías, entre otros, sino que, en su apuesta por las Humanidades Digitales, hace un uso pro-activo de un blog y de unos perfiles en las redes sociales que, aparentemente, nos hacen contactar con el lector virtual de manera eficaz. Con el objetivo de seguir mejorando, a través del presente trabajo se analizan qué temas han suscitado un mayor interés entre los lectores de nuestras herramientas sociales, a través del estudio de las publicaciones de nuestra bitácora 2.0 y del reflejo de éstas en nuestra página en Facebook. La respuesta nos ayudará a conocer mejor los intereses y necesidades de información de los usuarios y, consecuentemente, podremos seguir perfeccionando los puentes digitales que hemos tendido entre ellos y la Historia y del Patrimonio Naval y Marítimo.
\end{abstract}

Palabras clave: historia, patrimonio naval y maritimo, difusión, blog, Facebook, Pinake, Cátedra de Historia Naval, humanidades digitales

\begin{abstract}
The Naval History Chair, driven by the Spanish Navy and the University of Murcia, it has among its objectives to investigate and disseminate the History and the Naval and Maritime Heritage. In order to bring scientific knowledge and information about these themes to the user, our institution not only uses the classic communication channels such as articles and monographs, among others, but, in its commitment to the Digital Humanities, it makes a proactive use of a blog and a social networks profiles which, apparently, make us contact effectively with the virtual reader. In order that we continue improving, through this work, we analyze what issues have aroused greater interest among the readers of our social tolos, through the study of the publications of our blog and the reflection of these in our Facebook page. The answer will help us to better understand the interests and information needs of the users, as a result, we'll can further devolpt the digital bridges that we have layed between the and the History and the Naval and Maritime Heritage.
\end{abstract}

Keywords: History, Naval and Maritime Heritage, Dissemination, Blog, Facebook, Pinake, Naval History Chair, Digital Humanities

\section{Introducción y objetivos}

$\mathrm{E}$ 1 día 2 de diciembre de 2010 se firmó en Madrid un Convenio para fomentar, desarrollar y difundir los conocimientos sobre Historia, Patrimonio y Cultura Marítima a través de la creación de la primera Cátedra de Historia Naval2, de carácter interinstucional, impulsada por la Armada Española y la Universidad de Murcia.

\footnotetext{
1 www.um.es/catedranaval Este trabajo es uno de los resultados de los Proyectos I+D+I financiados por la Fundación Séneca Agencia Regional de Ciencia y Tecnología denominados ARGOS II, 11936/PHCS/09, y NAUTICUM, 19496/PI/14.

2 Página web $<$ http://www.um.es/catedranaval/ $>$.
}

Revista Internacional de Ciencias Humanas

Volumen 5, Número 2, <http://lascienciashumanas.com>, ISSN 2530-4526

(C) Global Knowledge Academics. Lorena Martínez Solís et al.

Todos los derechos reservados. Permisos: soporte@gkacademics.com 
Una de las actividades específicas que se llevan a cabo en el marco de la Cátedra es el Máster en Historia y Patrimonio $\mathrm{Naval}^{3}$, que se imparte desde el curso 2013/2014 por la Universidad de Murcia y en estrecha colaboración con la Armada. Además, esta institución lleva a cabo anualmente una programación, con la finalidad de desarrollar las siguientes actividades:

- Impartir seminarios o ciclos de conferencias relacionados con la Historia, Cultura y Patrimonio Naval.

- Realizar investigaciones en el área de Historia, Documentación y Patrimonio Naval, así como el estudio, descripción y difusión de fondos históricos que se conservan en los distintos archivos, museos y bibliotecas de la Armada.

- Promover la realización de tesis doctorales con los fondos históricos de la Armada.

- Potenciar y desarrollar todos aquellos aspectos relacionados con las ciencias auxiliares de la Historia Naval, a través de la realización de cursos de especialización.

- Difundir la Historia y el Patrimonio Naval a través de la creación y promoción de publicaciones en colecciones temáticas.

En relación con este último aspecto, el de la difusión, se debe precisar que la Cátedra, en su afán por acercar la Historia y el Patrimonio Naval al ciudadano, no se limita a transmitir la información y sus resultados de investigación a través de los medios tradicionales (ponencias, artículos y monografías), por otra parte necesarios y efectivos, sino que apuesta además por la difusión a través de las nuevas tecnologías.

El equipo humano que genera, da forma e impulsa las citadas actividades de esta institución, está formado principalmente por historiadores y documentalistas. Tanto unos como otros somos plenamente conscientes de que es necesario tender puentes entre los investigadores y la tecnología, y que la actitud de buscar al usuario, esté donde esté, siempre es básica. Entendemos que esta filosofía o modelo de trabajo, bien podría enmarcarse en lo que se conoce como Humanidades Digitales.

Es por ello que hemos puesto en marcha una serie de herramientas de las enmarcadas en la web social. Concretamente, la Cátedra dispone de un blog denominado Pinake (figura 1), así como de perfiles en las redes sociales más importantes.

Figura 1: Página de inicio blog Pinake

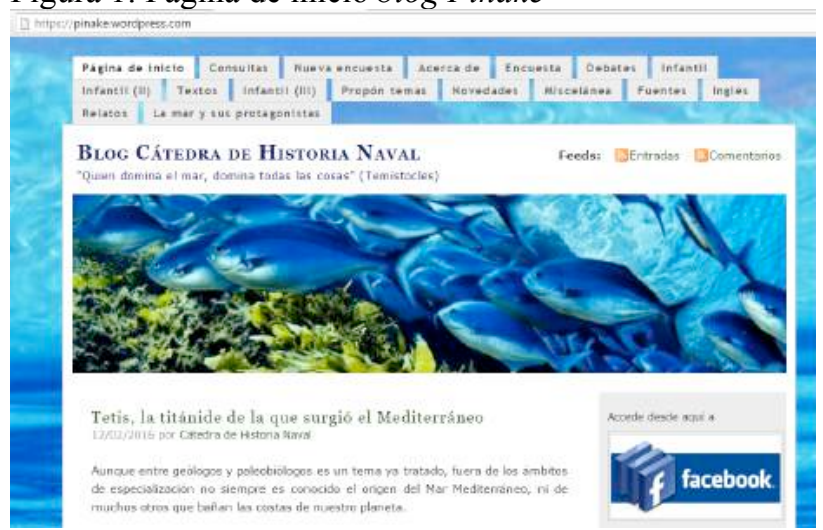

Fuente: Elaboración propia a partir de una captura de pantalla de la página de inicio del blog Pinake, disponible en http://pinake.wordpress.com/.

El objetivo básico del citado blog es divulgar el importante Patrimonio tangible (objetos históricos que evidencian las actividades realizadas en el pasado) y también el intangible, igual de importante, que incluye manifestaciones y productos resultados de la actividad de los seres humanos, pero que no se han recogido en soporte alguno (por ejemplo la lengua marinera mediterránea). Para ello

\footnotetext{
${ }^{3}$ Web del Máster $<$ http://mastia.wordpress.com/>

${ }^{4}$ Disponible en $<\underline{\text { http://pinake.wordpress.com } />}$.
} 
se ofrecen textos de interés sobre materias relacionadas con la Historia Naval y Marítima que se renuevan cada 6 o 7 días.

Los contenidos del blog se complementan con los de los perfiles de la Cátedra en las redes sociales. Aunque se dispone de Facebook, Twitter, Tuenti y Google+, en la actualidad el que más seguidores tiene es el primero de ellos.

La razón de ser de la página en Facebook ${ }^{5}$ (figura 2) de nuestra institución es dar a conocer diariamente, noticias, efemérides, hechos curiosos, entre otros, relacionados con la Historia y el Patrimonio Naval. Es por ello que en ocasiones este perfil actúa como "relaciones públicas" dando a conocer las publicaciones de nuestro blog Pinake.

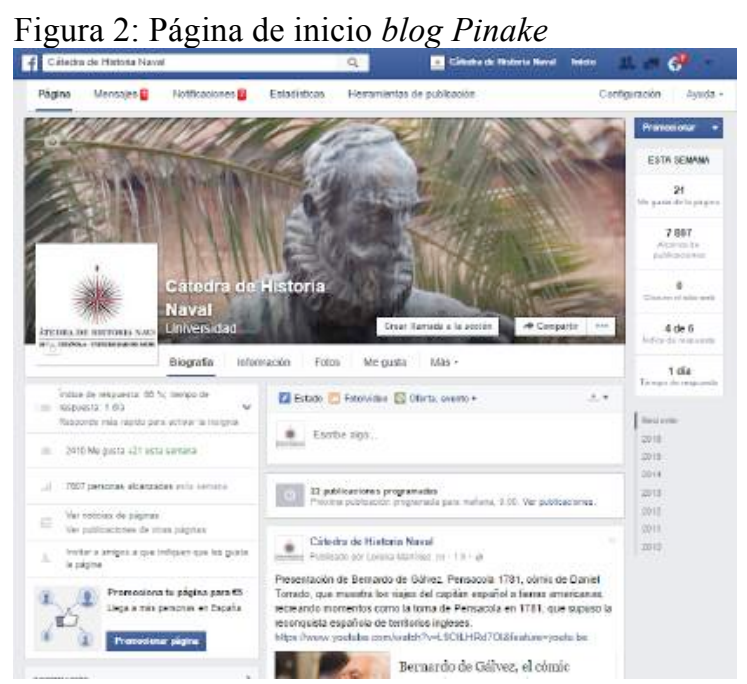

Fuente: Elaboración propia a partir de una captura de pantalla de la página de inicio del blog Pinake, disponible en <http://pinake.wordpress.com/>.

Sin entrar demasiado en materia, podemos decir que a día de hoy, y después de casi cuatro años desde su puesta en marcha, el blog ha recibido cerca de 500 mil visitas y el perfil en Facebook tiene casi 1.700 seguidores.

Dado este aparente éxito, el objetivo del presente trabajo es analizar qué temas han suscitado un mayor interés en los usuarios de nuestras herramientas sociales, a través del estudio del número de visitas recibidas en las publicaciones de nuestra bitácora 2.0, y de su reflejo en nuestra página en Facebook. La respuesta nos ayudará a conocer mejor sus intereses y necesidades de información y, consecuentemente, podremos seguir perfeccionando los puentes digitales tendidos entre ellos y la Historia y del Patrimonio Naval y Marítimo.

\section{Hacia las Humanidades Digitales: breve estado de la cuestión}

Bajo la denominación de Humanidades Digitales (HD) últimamente están apareciendo una serie de iniciativas encaminadas a propiciar el uso habitual de las tecnologías en las ramas más cualitativas de la investigación. Con relativa frecuencia se habla de incorporar las Humanidades en las tecnologías, variando el orden de prelación: las tecnologías son los instrumentos, el objetivo último es apoyar la docencia y la investigación que se hace en las disciplinas humanísticas, y no al contrario.

Partiendo de este cambio en el orden, podemos empezar a enumerar faltas de sintonía y disfunciones variadas ocurridas entre profesionales acostumbrados a que el ordenador y sus contenidos son el producto último (técnicos informáticos) e investigadores habituados a que la palabra escrita en papel, o verbalizada de manera presencial, sean la base de la comunicación.

\footnotetext{
${ }^{5}$ Disponible en $<$ https://www.facebook.com/catedranaval $>$.
} 
En este sentido el término "Humanidades Digitales" se está afianzando en el uso frente a otros como Humanities Computing (Leibrandt, 2006), Recursos Digitales para las Humanidades, Informática para las Humanidades o en las Humanidades, Informática Cultural,... Rodríguez Yunta en una búsqueda en dos bases de datos internacionales (Scopus y Web of Science) con las palabras "Digital Humanities" en el título, resumen o palabras clave, muestra que es un concepto de uso creciente, especialmente en los dos últimos años (Rodríguez Yunta, 2012).

La mayor parte de las iniciativas de apoyo a la generación de recursos digitales sobre Humanidades surgen en el ámbito anglosajón, en países como Estados Unidos, Reino Unido y Canadá (Galina-Russell, 2011; Leibrandt, 2006), y terminan generando asociaciones (Alliance of Digital Humanities Organizations), fundaciones, centros o departamentos universitarios específicos (Digital Humanities Centres), cursos de posgrado, congresos o reuniones (THATCamp), blogs y revistas especializadas (Digital Humanities Quarterly) (Rodríguez Yunta, 2002).

En el "Manifiesto por unas Humanidades Digitales" (Dacos, 2011), redactado en mayo de 2010 en París, se definen éstas como una "transdisciplina" portadora de los métodos, dispositivos y perspectivas heurísticas relacionadas con procesos de digitalización en el campo de las Ciencias Humanas y Sociales. Más allá de la definición, este documento reclama el libre acceso a datos y metadatos, la libre circulación de métodos y resultados de la investigación y la creación de títulos específicos en HD (Rodríguez Yunta, 2002) y llaman a la construcción de ciberestructuras evolutivas que respondan a necesidades reales. Estas ciberinfraestructuras se irán construyendo de forma interactiva al apoyarse en la comprobación de métodos y aproximaciones llevados a cabo dentro de las comunidades de investigación (artículo 14) ${ }^{6}$ (Dacos, 2011).

Otro manifiesto generado también en Estados Unidos, en el Center for Digital Humanities de la Universidad de UCLA (Presner, 2009) incide en reclamar la reflexión colectiva sobre el conocimiento y la cultura digital y el compromiso con el acceso abierto.

Casi todos los artículos que se han escrito sobre el tema terminan recomendando el trabajo interdisciplinar entre especialistas del campo de la Documentación y los investigadores del área de las Humanidades, como ocurre en nuestro grupo de investigación, con amplitud de miras y horizontes abiertos (Siemenes et al., 2011: pp. 335-348). Mantienen que el espacio es todavía impreciso. Sin embargo, no hay duda que está en expansión (un buen ejemplo de ello es la arqueología virtual, entre los que se puede citar Digitalmed Centro Mediterráneo de Innovación Digital en Patrimonio Cultural $\left.{ }^{7}\right)$, aunque no estén enteramente claro cuál es su campo de acción y cómo estarán estructuradas (Svensson, 2010).

\section{Metodología}

Para llevar a cabo nuestro estudio seleccionamos la información disponible de todas y cada una de las entradas publicadas en el blog Pinake desde que se puso en marcha, mayo de 2011, hasta la fecha. Estos datos estaban disponibles en el panel de estadísticas de cada una de las herramientas (bitácora y perfil social). En total se analizaron 250 publicaciones ( 250 entradas en el blog y sus 250 réplicas en Facebook).

Con objeto de poder gestionar adecuadamente la información se generó una base de datos con Microsoft Access. Para trabajar la parte del blog se incluyeron los campos: categorías temáticas (definidas por nosotros en la bitácora a la hora de publicar una entrada) y número de visitas (que ha recibido la entrada desde que se publicó). Para el perfil en Facebook se utilizó el campo alcance (número de veces que ha sido vista la entrada).

\footnotetext{
6 También el Artículo 13 manifiesta "Deseamos participar en la definición y difusión de prácticas aconsejables en relación con necesidades disciplinarias y transdisciplinarias debidamente identificadas, evolutivas y derivadas de un debate y luego de un consenso por lograr en el marco de las mencionadas comunidades. La apertura fundamental de las Humanidades Digitales conlleva sin embargo una aproximación pragmática de los protocolos y de las visiones que preserva el derecho a la coexistencia de métodos distintos que hasta llegan a competir entre sí, para el mayor provecho de la reflexión y de las prácticas" (Dacos, 2011).

${ }^{7}$ Digitalmed. Disponible en $<$ http://goo.gl/6YdcmN $>$.
} 
Una vez diseñada la base de datos, analizamos y comparamos el número de visitas que recibió cada ítem en cada una de las herramientas (blog y Facebook). Primero observamos el total de accesos/visualizaciones recibidas por entrada a lo largo de todo el período y después el número de visitas obtenidas ordenadas cronológicamente por su fecha de publicación.

Finalmente, observamos cuáles han sido las 25 entradas (hemos considerado que es una cantidad suficiente y representativa) que han recibido más visitas tanto en la bitácora como en el perfil social, así como las categorías temáticas a las que pertenecen, y finalmente comparamos el resultado obtenido en cada una de las herramientas 2.0.

\section{Resultados}

Se observa que las 250 publicaciones reciben en el blog una media de 815 visitas, pero de manera específica una entrada recibe más de 13 mil visitas $(0,4 \%)$, otra más de 6 mil $(0,4 \%)$, tres reciben más de 5 mil (1,2\%), dos más de 4 mil $(0,8 \%)$, seis más de 3 mil $(2,4 \%)$, nueve más de 2 mil $(3,6 \%)$ y 33 más de mil $(13,2 \%)$. El resto (198) recibe menos de mil $(79,2 \%)$ decreciendo de manera muy acusada. (Figuras 3 y 4 ).

Figuras 3 y 4: Visitas que reciben las entradas en el blog, en total y ordenadas cronológicamente

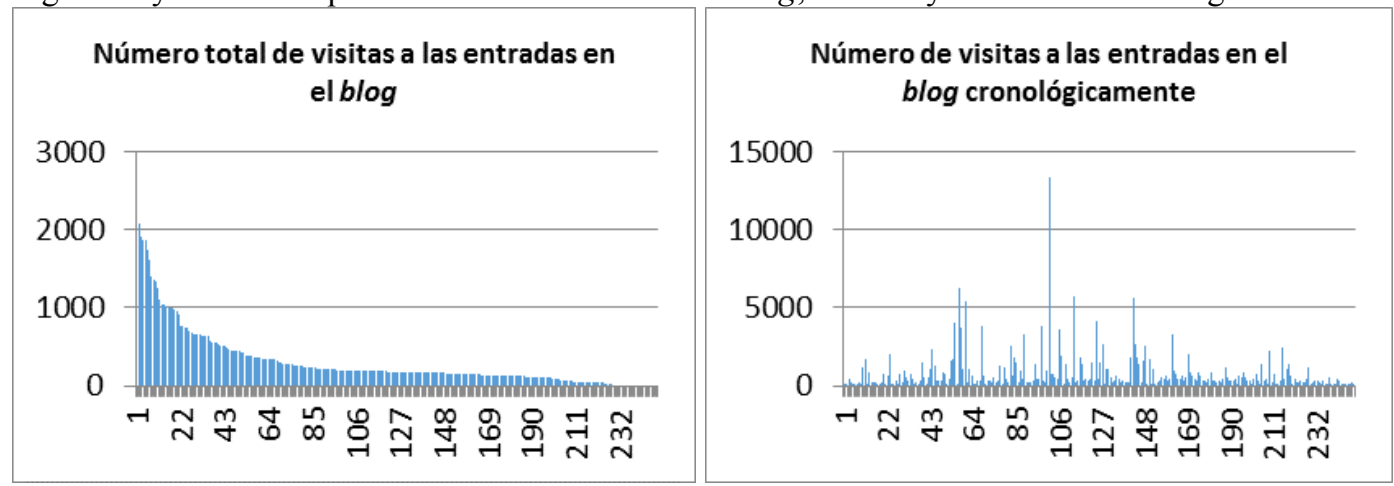

Fuente: Elaboración propia.

En Facebook, los 250 anuncios de las entradas del blog reciben una media de 310 visitas. De manera específica, dos fueron vistos más de 2 mil veces $(0,8 \%), 15$ más de mil $(6 \%)$, y el resto (236) recibe menos de mil $(94,4 \%)$ decreciendo de manera gradual. (Figura 5 y 6 ).

Figura 5 y 6: Visitas que reciben las entradas en Facebook, en total y ordenadas cronológicamente
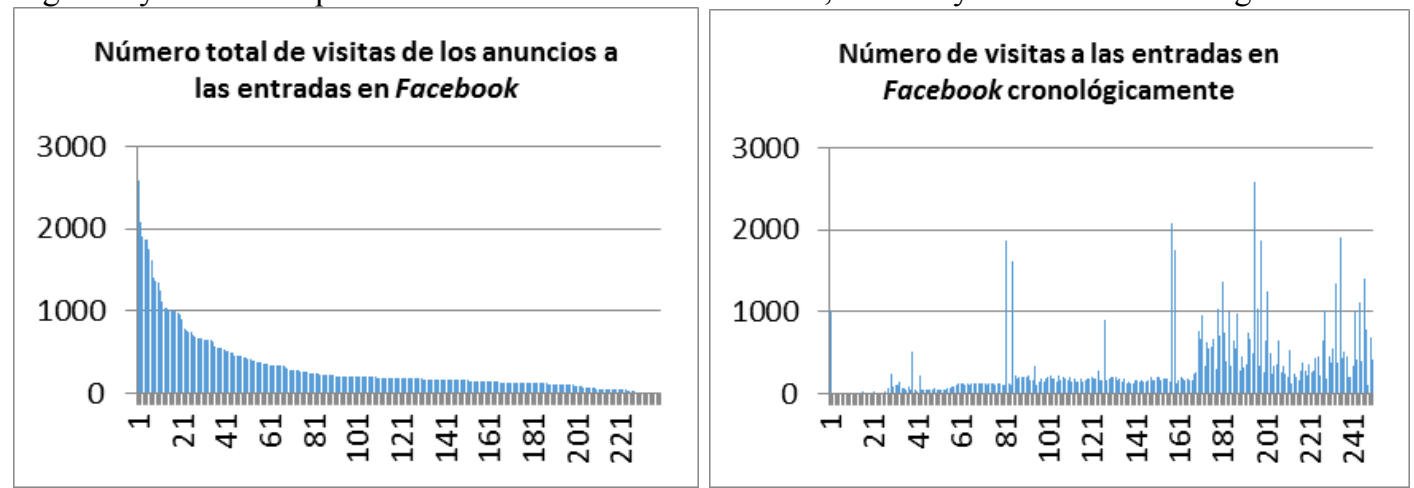

Fuente: Elaboración propia. 
Dentro del $b \log$, las 25 entradas que han recibido más visitas han sido las siguientes (figura 7):

Figura 7: Entradas que han recibido más visitas en el blog

\begin{tabular}{|c|c|c|c|}
\hline & $\begin{array}{l}\mathbf{N}^{0} \text { de } \\
\text { visitas }\end{array}$ & Nombre de la entrada & Categorías \\
\hline 1 & 13.369 & $\begin{array}{l}\text { Los "caballitos de totora" del lago } \\
\text { Titicaca }\end{array}$ & General, jóvenes historiadores \\
\hline 2 & 6.268 & $\begin{array}{l}\text { Una princesa rusa asesinada en la } \\
\text { isla del Barón }\end{array}$ & Leyendas y mitos marineros \\
\hline 3 & 5.727 & El viaje que inspiró a Darwin & Jóvenes historiadores \\
\hline 4 & 5.590 & $\begin{array}{l}\text { Navíos españoles del siglo XVIII } \\
\text { (I) }\end{array}$ & $\begin{array}{l}\mathrm{H}^{\mathrm{a}} \text { Construcción Naval, Historia Naval, Patrimonio } \\
\text { Naval }\end{array}$ \\
\hline 5 & 5.465 & $\begin{array}{l}\text { Isabel Grameson: la última mujer } \\
\text { que recorrió el Amazonas }\end{array}$ & Historia Marítima \\
\hline 6 & 4.137 & $\begin{array}{l}\text { Faro de Cabos de Palos, Cartagena } \\
\text { (España) }\end{array}$ & Faros en la Historia \\
\hline 7 & 4.062 & $\begin{array}{l}\text { Comunicándose entre barcos } \sin \\
\text { tecnología punta }\end{array}$ & Historia Marítima, Historia Naval \\
\hline 8 & 3.808 & $\begin{array}{l}\text { La leyenda del marinero y la sirena } \\
\left.\text { ( } 1^{\text {a }} \text { parte }\right)\end{array}$ & Leyendas y mitos marineros \\
\hline 9 & 3.808 & Museo Marítimo de Creta & Museos Navales \\
\hline 10 & 3.742 & $\begin{array}{l}\text { Construcción Naval en la Historia: } \\
\text { vocabulario }\end{array}$ & $\mathrm{H}^{\mathrm{a}}$ Construcción naval \\
\hline 11 & 3.649 & $\begin{array}{l}\text { "Las reglas del diablo": el código } \\
\text { de los piratas }\end{array}$ & General, jóvenes historiadores \\
\hline 12 & 3.301 & $\begin{array}{l}\text { Hipona, la ciudad marítima de San } \\
\text { Agustín }\end{array}$ & Ciudades marítimas, Historia Marítima \\
\hline 13 & 3.258 & $\begin{array}{l}\text { Ushuaia: los museos del fin del } \\
\text { mundo }\end{array}$ & Museos Navales \\
\hline 14 & 2.691 & $\begin{array}{l}\text { Navíos españoles del siglo XVIII } \\
\text { (II) }\end{array}$ & $\begin{array}{l}\mathrm{H}^{\mathrm{a}} \text { Construcción Naval, Historia Marítima, Historia } \\
\text { Naval }\end{array}$ \\
\hline 15 & 2.634 & $\begin{array}{l}\text { El ojo de Horus: protección y } \\
\text { defensa en el mar }\end{array}$ & $\begin{array}{l}\text { General, Historia Marítima, Historia Naval, leyendas } \\
\text { y mitos marineros, Patrimonio Naval }\end{array}$ \\
\hline 16 & 2.596 & $\begin{array}{l}\text { El concepto de "sirena" a lo largo } \\
\text { de la historia }\end{array}$ & Leyendas y mitos marineros \\
\hline 17 & 2.574 & $\begin{array}{l}\text { Navíos españoles del siglo XVIII } \\
\text { (VI) }\end{array}$ & $\begin{array}{l}\mathrm{H}^{\mathrm{a}} \text { Construcción Naval, Historia Marítima, Historia } \\
\text { Naval }\end{array}$ \\
\hline 18 & 2.494 & Sorolla, el pintor del mar & General, Museos Navales, Patrimonio Naval \\
\hline 19 & 2.378 & $\begin{array}{l}\text { Los nombres de los vientos mari- } \\
\text { nos en la Historia (I) }\end{array}$ & Historia Marítima \\
\hline 20 & 2.275 & $\begin{array}{l}\text { Faro de Cabo de Gata: entre le- } \\
\text { yendas de piratas, naufragios y } \\
\text { sirenas }\end{array}$ & $\begin{array}{l}\text { Faros en la Historia, General, Historia Marítima, } \\
\text { Historia Naval, leyendas y mitos marineros, pecios }\end{array}$ \\
\hline 21 & 2.073 & Siracusa, la ciudad griega de Italia & $\begin{array}{l}\text { Ciudades marítimas, Historia Marítima, Historia } \\
\text { Naval, Patrimonio Naval }\end{array}$ \\
\hline 22 & 2.042 & El faro de Alejandría & Faros en la Historia \\
\hline 23 & 1.965 & $\begin{array}{l}\text { Oro americano en barcos españo- } \\
\text { les: el pecio del "Juncal" }\end{array}$ & Historia Marítima, Historia Naval \\
\hline 24 & 1.816 & Farallones de Capri (Italia) & Farallones: los ojos del mar \\
\hline 25 & 1.814 & $\begin{array}{l}\text { Un manuscrito básico para enten- } \\
\text { der la construcción naval española }\end{array}$ & $\begin{array}{l}\text { Archivos y bibliotecas, } \mathrm{H}^{\mathrm{a}} \text { Construcción Naval, } \\
\text { Historia Naval, Museos Navales, Patrimonio Naval, } \\
\text { un mar de libros digitalizados }\end{array}$ \\
\hline
\end{tabular}

Fuente: Elaboración propia. 
En base a la tabla anterior, los temas más consultados en el blog son los siguientes (figura 8):

Figura 8: Temas más consultados en el blog

\begin{tabular}{|clcl|}
$\begin{array}{c}\mathbf{N}^{\mathbf{0}} \text { de entra- } \\
\text { das }\end{array}$ & \multicolumn{1}{c|}{ Categorías } & $\begin{array}{c}\mathbf{N}^{\mathbf{0}} \text { de entra- } \\
\text { das }\end{array}$ & \multicolumn{1}{c|}{ Categorías } \\
\hline $\mathbf{1 0}$ & Historia Marítima & 3 & Faros en la Historia \\
\hline $\mathbf{9}$ & Historia Naval & 2 & Jóvenes historiadores \\
\hline $\mathbf{6}$ & $\begin{array}{l}\text { Leyendas y mitos marine- } \\
\text { ros }\end{array}$ & 1 & Archivos y bibliotecas \\
\hline $\mathbf{5}$ & $\mathrm{H}^{\text {a } \text { Construcción Naval }}$ & 1 & Ciudades marítimas \\
\hline $\mathbf{5}$ & Patrimonio Naval & 1 & $\begin{array}{l}\text { Farallones: los ojos del mar Ciudades } \\
\text { marítimas }\end{array}$ \\
\hline $\mathbf{4}$ & General & 1 & Pecios \\
\hline $\mathbf{4}$ & Museos Navales & 1 & Un mar de libros digitalizados \\
\hline
\end{tabular}

Fuente: Elaboración propia.

En Facebook, las 25 publicaciones más vistas han sido las siguientes (figura 9).

Figura 9: Entradas que han recibido más visitas en Facebook

\begin{tabular}{|c|c|c|c|}
\hline & $\begin{array}{l}\mathbf{N}^{0} \text { de } \\
\text { visitas }\end{array}$ & Nombre de la entrada & Categorías \\
\hline 1 & 2580 & $\begin{array}{l}\text { Una fuente para conocer el Medi- } \\
\text { terráneo Medieval }\end{array}$ & $\begin{array}{l}\text { Archivos y bibliotecas, ciudades marítimas, Historia } \\
\text { Marítima, Historia Naval, Patrimonio Naval, un mar } \\
\text { de libros digitalizados }\end{array}$ \\
\hline 2 & 2086 & $\begin{array}{l}\text { Los libros Generales de las Gale- } \\
\text { ras de España }\end{array}$ & $\begin{array}{l}\text { El mundo de las galeras, Historia Marítima, Historia } \\
\text { Naval, Patrimonio Naval, un mar de libros digitaliza- } \\
\text { dos }\end{array}$ \\
\hline 3 & 1917 & $\begin{array}{l}\text { El camino español a Flandes } \\
\text { partía desde Cartagena }\end{array}$ & $\begin{array}{l}\text { Ciudades marítimas, el mundo de las galeras, Historia } \\
\text { Marítima, Historia Naval, puertos con historia }\end{array}$ \\
\hline 4 & 1875 & $\begin{array}{l}\text { El concepto de "sirena" a lo largo } \\
\text { de la historia }\end{array}$ & Leyendas y mitos marineros \\
\hline 5 & 1874 & $\begin{array}{l}\text { Gabriel Ciscar, otro genio incom- } \\
\text { prendido }\end{array}$ & Guardiamarinas, Historia Marítima, Historia Naval \\
\hline 6 & 1749 & $\begin{array}{l}\text { Los inicios de la búsqueda de } \\
\text { pecios }\end{array}$ & General, Historia Marítima, Patrimonio Naval, pecios \\
\hline 7 & 1624 & Ornato y distintivo en las galeras & El mundo de las galeras \\
\hline 8 & 1411 & $\begin{array}{l}\text { La Historia Marítima pierde a } \\
\text { Casado Soto }\end{array}$ & $\begin{array}{l}\text { General, Historia Marítima, Historia Naval, Museos } \\
\text { Navales, Patrimonio Naval }\end{array}$ \\
\hline 9 & 1365 & $\begin{array}{l}\text { Compendio de navegación de } \\
\text { Jorge Juan }\end{array}$ & $\begin{array}{l}\text { Archivos y bibliotecas, guardiamarinas, } \mathrm{H}^{\mathrm{a}} \text { Construc- } \\
\text { ción Naval, Historia Marítima, Historia Naval, mari- } \\
\text { nos ilustrados, Patrimonio Naval }\end{array}$ \\
\hline 10 & 1344 & $\begin{array}{l}\text { Las fuentes del Nilo las descubrió } \\
\text { un misionero español }\end{array}$ & Grandes viajeros \\
\hline 11 & 1252 & $\begin{array}{l}\text { Las antiguas ciudades mediterrá- } \\
\text { neas en Internet }\end{array}$ & $\begin{array}{l}\text { Ciudades marítimas, Historia Marítima, Historia } \\
\text { Naval, Patrimonio Naval, publicaciones antiguas, un } \\
\text { mar de libros digitalizados }\end{array}$ \\
\hline 12 & 1109 & $\begin{array}{l}\text { Baterías de costa gaditanas duran- } \\
\text { te la Guerra de la Independencia } \\
\text { (II) }\end{array}$ & $\begin{array}{l}\text { Ciudades marítimas, general, Historia Marítima, } \\
\text { Historia Naval, puertos con historia }\end{array}$ \\
\hline 13 & 1040 & $\begin{array}{l}\text { Academia de Guardiamarinas de } \\
\text { Cartagena (1777-1824) }\end{array}$ & Guardiamarinas, Historia Marítima, Historia Naval \\
\hline 14 & 1035 & $\begin{array}{l}\text { Nace la colección Cátedra de } \\
\text { Historia Naval }\end{array}$ & $\begin{array}{l}\text { Archivos y bibliotecas, general, Historia Marítima, } \\
\text { Historia Naval, libros de interés, Patrimonio Naval }\end{array}$ \\
\hline 15 & 1015 & In memorian: Ciudades & Ciudades marítimas, general \\
\hline
\end{tabular}




\begin{tabular}{|c|c|c|c|}
\hline & & barco, de Gabo & \\
\hline 16 & 1010 & 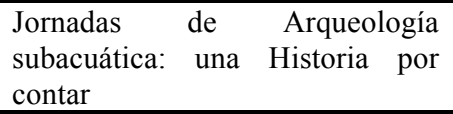 & $\begin{array}{l}\text { General, Historia Marítima, Historia Naval, Patrimo- } \\
\text { nio Naval, pecios }\end{array}$ \\
\hline 17 & 1006 & $\begin{array}{l}\text { La mujer que sirvió en la fragata } \\
\text { Mercedes: Antonio } \mathrm{M}^{\mathrm{a}} \text { de Soto }\end{array}$ & $\begin{array}{l}\text { General, Historia Marítima, Historia Naval, historias } \\
\text { que nos han contado }\end{array}$ \\
\hline 18 & 998 & $\begin{array}{l}\text { Un mar de libros digitalizados } \\
\text { (II): Hispana }\end{array}$ & $\begin{array}{l}\text { Historia Marítima, Historia Naval, Patrimonio Naval, } \\
\text { un mar de libros digitalizados }\end{array}$ \\
\hline 19 & 970 & Biblioteca Virtual de Defensa & $\begin{array}{l}\text { Archivos y Bibliotecas, Historia Marítima, Historia } \\
\text { Naval, Patrimonio Naval, publicaciones antiguas, un } \\
\text { mar de libros digitalizados }\end{array}$ \\
\hline 20 & 960 & $\begin{array}{l}\text { Guardiamarinas del s. XVIII: } \\
\text { oficiales e intelectuales (I) }\end{array}$ & Guardiamarinas, Historia Marítima, Historia Naval \\
\hline 21 & 906 & $\begin{array}{l}\text { El ojo de Horus: protección y } \\
\text { defensa en el mar }\end{array}$ & $\begin{array}{l}\text { General, Historia Marítima, Historia Naval, leyendas } \\
\text { y mitos marineros, Patrimonio Naval }\end{array}$ \\
\hline 22 & 777 & $\begin{array}{l}1^{\mathrm{a}} \text { promoción de expertos en } \\
\text { Historia y Patrimonio Naval }\end{array}$ & $\begin{array}{l}\text { General, Historia Marítima, Historia Naval, jóvenes } \\
\text { historiadores }\end{array}$ \\
\hline 23 & 761 & $\begin{array}{l}\text { La fragata Mercedes: un buque de } \\
\text { estado }\end{array}$ & General, museos navales, Patrimonio Naval \\
\hline 24 & 755 & $\begin{array}{l}\text { Los grafiti navales del Palacio de } \\
\text { Ambel (Zaragoza) }\end{array}$ & $\begin{array}{l}\text { El mundo de las galeras, general, } \mathrm{H}^{\mathrm{a}} \text { Construcción } \\
\text { Naval, Historia Marítima, Historia Naval, Patrimonio } \\
\text { Naval }\end{array}$ \\
\hline 25 & 745 & $\begin{array}{l}\text { Europeana, patrimonio al alcance } \\
\text { de tod@s }\end{array}$ & $\begin{array}{l}\text { Archivos y bibliotecas, museos navales, Patrimonio } \\
\text { Naval, un mar de libros digitalizados }\end{array}$ \\
\hline
\end{tabular}

Fuente: Elaboración propia.

Teniendo en cuenta la figura anterior, los temas a los que pertenecen las publicaciones en Facebook son los siguientes (figura 10).

Figura 10: Temas a los que pertenecen las entradas de Facebook

\begin{tabular}{|clcl|}
\hline$N^{\mathbf{0}}$ de entradas & \multicolumn{1}{c|}{ Categorías } & $\mathbf{N}^{\mathbf{0}}$ de entradas & \multicolumn{1}{c|}{ Categorías } \\
\hline $\mathbf{1 9}$ & Historia Marítima & 2 & $\mathrm{H}^{\text {a }}$ Construcción Naval \\
\hline $\mathbf{1 8}$ & Historia Naval & 2 & Leyendas y mitos marineros \\
\hline $\mathbf{1 4}$ & Patrimonio Naval & 2 & Pecios \\
\hline $\mathbf{1 1}$ & General & 2 & Publicaciones antiguas \\
\hline $\mathbf{6}$ & Un mar de libros digitalizados & 2 & Puertos con historia \\
\hline $\mathbf{5}$ & Archivos y bibliotecas & 1 & Grandes viajeros \\
\hline $\mathbf{5}$ & Ciudades marítimas & 1 & Historias que nos han contado \\
\hline $\mathbf{4}$ & El mundo de las galeras & 1 & Jóvenes historiadores \\
\hline $\mathbf{4}$ & Guardiamarinas & 1 & Libros de interés \\
\hline $\mathbf{3}$ & Museos navales & 1 & Marinos ilustrados \\
\hline
\end{tabular}

Fuente: Elaboración propia.

\section{Conclusiones}

La primera conclusión alcanzada en cuanto al número total de visitas recibidas por una entrada, es que éste es dispar. En el blog las entradas reciben más lectores pero su número decrece de manera muy acusada, es decir, unas publicaciones reciben muchas y otras muy pocas. En Facebook, sin embargo, obtienen menos visitas pero son más regulares. Creemos que el número de visitas que consiguen las publicaciones en la red social son más homogéneas, en general, porque en esta aplicación cuando un usuario sigue un perfil con regularidad, en su timeline o panel de novedades (página de inicio) le aparecen sistemáticamente las entradas de la página a la que sigue. Es decir, no es un 
acto voluntario, como sí lo es en el blog acudir a leer el artículo. Por lo tanto, lo normal es que si una página cuenta con $\mathrm{X}$ seguidores leales, todos ellos vean las publicaciones.

Por otro lado, si ordenamos las entradas por fecha publicación, observamos que el número de visitas en el blog crece hasta casi la mitad del periodo para después comenzar a decrecer. Creemos que esto es debido al cambio en la regularidad de la publicación de las entradas: en un principio se posteaba cada 3 o 4 días pero más tarde, por falta de recursos, principalmente de tiempo, y con objeto de no menguar la calidad de las publicaciones, se comenzó a publicar una vez a la semana. Puede que ello haya contribuido a que disminuya el número de visitas.

En Facebook, utilizando el mismo sistema de ordenación, podemos ver que el número de visitas aumenta progresivamente. Sin duda, este incremento anda de la mano con el crecimiento exponencial de seguidores en nuestro perfil social.

Centrándonos en los contenidos, de las 25 entradas más consultadas tanto en el blog (2.634 visitas) como en Facebook (906 visitas), tan sólo una coincide en ambas aplicaciones, la publicación titulada: "El ojo de Horus: protección y defensa en el mar".

En cuanto a los temas más consultados, los cinco más leídos en el blog han sido: Historia Marítima, Historia Naval, leyendas y mitos marineros, $\mathrm{H}^{\mathrm{a}}$ de la Construcción Naval y Patrimonio Naval. Por su parte, las categorías temáticas a las que pertenecen las publicaciones en Facebook son: Historia Marítima, Historia Naval, Patrimonio Naval, general y un mar de libros digitalizados.

Observamos que, aparte de las entradas relacionadas con la Historia Naval, la Historia Marítima, temática general, y Patrimonio Naval, categorías por otra parte de tipo genérico (al fin y al cabo es un blog en el que todas las publicaciones tienen que ver con Historia y/o Patrimonio Naval y Marítimo), las más visitadas son las que versan sobre leyendas y mitos y marineros, $\mathrm{H}^{\mathrm{a}}$ construcción naval y sobre libros digitalizados. Este último tema, estrechamente relacionado con los repositorios y las humanidades digitales.

Finalmente, al interrelacionar los datos obtenidos de las entradas más vistas en el blog con su repercusión en Facebook, observamos que, en los últimos tiempos (conforme hemos ido ganando mayor número de seguidores en el perfil 2.0), algunas de las publicaciones de la bitácora que han sido más visitadas, son también algunas de las favoritas en la red social.

En otras palabras, el anunciar los nuevos post en la red social genera una corriente de lectores hacia el blog. Pero estas dos herramientas tienen un número de seguidores diferentes, ya que hay lectores que prefieren la rapidez e instantaneidad de Facebook, mientras que otras eligen leer entradas del blog que tienen mucho más calado y profundidad.

Este tipo de herramientas 2.0 no permiten que se haga una identificación de los seguidores, pero posiblemente las edades de los que prefieren la herramienta social sean menores que los lectores de la bitácora digital. 


\section{REFERENCIAS}

Dacos, M. (2011). Manifiesto por unas Humanidades Digitales. Thatcamp. User generated "uncoference" on digital humanities [en línea]. [Consulta: 15-12-2012]. Disponible en $<$ http://tcp.hypotheses.org/487>.

Galina-Russell, I. (2011). ¿Qué son las Humanidades Digitales? Revista Digital Universitaria [en línea], 12(7). [Consulta: 15-12-2012]. Disponible en:

$<$ http://www.revista.unam.mx/vol.12/num7/art68/>.

Leibrandt, I. (2006). Humanidades digitales, ¿ciencia ficción o realidad inminente?. Espéculo. Revista de estudios literarios [en línea], (33). [Consulta: 15-12-2012]. Disponible en: $<$ http://www.ucm.es/info/especulo/numero33/humadigi.html $>$.

Presner, T. (2009). Digital Humanities Manifesto 2.0 Launched. Digital Humanities [en línea]. [Consulta: 15-12-2012]. Disponible en $<$ http://www.toddpresner.com/?p=7 $>$.

Rodríguez Yunta, L. (2012). Las Humanidades Digitales, ¿una mera etiqueta o un campo por el que deben apostar las Ciencias de la Documentación? Notas ThinkEPI, 2013 [en línea]. [Consulta: 15-12-2012]. Disponible en: <http://goo.gl/irL16R>.

Siemenes, L. et al. (2011). A tale of two cities: implications of the similarities and differences in collaborative approaches within the digital libraries and digital humanities communities. Literary and linguistic computing [en linea], 26(3), 335-348. Disponible en: $<$ http://dx.doi.org/10.1093/1lc/fqr028>.

Svensson, P. (2010). The Landscape of Digital Humanities. Digital Humanities Quarterly [en linea], 4(1). [Consulta: 15-12-2012]. Disponible en:

$<$ http://digitalhumanities.org/dhq/vol/4/1/000080/000080.html $>$. 